\title{
Engineering of TMDC-OSC Hybrid Interfaces: The Thermodynamics of Unitary and Mixed Acene Monolayers on $\mathrm{MoS}_{2}{ }^{\dagger}$
}

Received 00th January 20xx, Accepted 00th January 20xx DOI: $10.1039 / x 0 x x 00000 x$

\author{
Stefan R. Kachel, $\ddagger^{\text {a }}$ Pierre-Martin Dombrowski, $\ddagger^{\mathrm{b}}$ Tobias Breuer, $^{\mathrm{b}}$ J. Michael Gottfried ${ }^{\mathrm{a}}$ and Gregor \\ Witte $* b$
}

\begin{abstract}
Hybrid systems of two-dimensional (2D) materials such as transition metal dichalcogenides (TMDCs) and organic semiconductors (OSCS) have become subject of great interest for future device architectures. Although OSC-TMDC hybrid systems have been used in first device demonstrations, the precise preparation of ultra-thin OSC films on TMDCs has not been addressed. Due to the weak van der Waals interaction between TMDCs and OSCs, this requires precise knowledge of the thermodynamics at hand. Here, we use temperature-programmed desorption (TPD) and Monte Carlo (MC) simulations of TPD traces to characterize the desorption kinetics of pentacene (PEN) and perfluoropentacene (PFP) on MoS 2 as a model system for OSCS on TMDCs. We show that the monolayers of PEN and PFP are thermally stabilized compared to their multilayers, which allows to prepare nominal monolayers by selective desorption of multilayers. This stabilization is, however, caused by entropy due to a high molecular mobility rather than an enhanced molecule-substrate bond. Consequently, the nominal monolayers are not densely packed films. Molecular mobility can be suppressed in mixed monolayers of PEN and PFP that, due to intermolecular attraction, form highly ordered films as shown by scanning tunneling microscopy. Although this reduces the entropic stabilization, the intermolecular attraction further stabilizes mixed films.
\end{abstract}

\section{Introduction}

Since the first successful isolation of graphene, interest in the field of two-dimensional materials has been continuously growing. ${ }^{1,2}$ Offering a wide range of charge transport properties ranging from insulators such as hexagonal boron nitride to semiconductors like transition metal dichalcogenides (TMDCs) to semi-metallic materials such as graphene, van der Waals ( $v d W$ ) bound hetero systems of 2D materials (2DMs) provide unprecedented prospects for device miniaturization in nextgeneration electronics. ${ }^{3-8}$ Besides the aspect of device miniaturization, the single-layer structure of these materials offers versatile possibilities for the development of flexible nanostructures with atomically sharp interfaces. ${ }^{9,10}$

A promising extension of the number of possible vdW hetero systems that retains the desired 2D structure and flexibility is provided by the combination of 2DMs with thin layers of organic semiconductors (OSCs) in organic-2DM hybrid systems. ${ }^{11,12}$ Since properties of OSCs are more easily tuneable through synthesis than those of 2DMs, such hybrid heterostructures can greatly facilitate device engineering. Moreover, OSCs can

\footnotetext{
a. Fachbereich Chemie, Philipps-Universität Marburg, Hans-Meerwein-Straße 4, 35032 Marburg, Germany.

b. Fachbereich Physik, Philipps-Universität Marburg, Renthof 7, 35032 Marburg, Germany. E-Mail: gregor.witte@physik.uni-marburg.de

+ Electronic Supplementary Information (ESI) available: Detailed information on the MC TPD simulation, Kelvin probe measurements of $\mathrm{MoS}_{2}$ and molecular films on $\mathrm{MoS}_{2}$, analysis of TPD traces, derivation of equations from transition state theory, structural analysis of STM data. See DOI: 10.1039/x0xx00000x

¥ These authors have contributed equally to this work.
}

complement 2DMs: While 2DMs generally have a higher charge carrier mobility, OSCs offer a high optical absorption efficiency, which renders the combination of OSCs with 2DMs particularly beneficial for optoelectronic applications such as photovoltaics. ${ }^{13}$ In fact, OSC-2DM hybrid systems have already been realized in photovoltaic devices ${ }^{14-17}$ and $p$-n junctions. ${ }^{16,18-}$ ${ }^{23}$ However, while theoretical concept studies focus on highly ordered OSC monolayers or single molecules on $2 \mathrm{DMs},{ }^{24,25}$ real hybrid heterosystems are usually restricted to OSC multilayers. This is because, unlike monolayers of 2DMs that are commercially available, the fabrication of true single-layer hybrid systems remains challenging as it requires highly precise dosing in molecular beam epitaxy.

A more scalable approach to the fabrication of molecular monolayers is that of selective desorption of multilayers that is commonly applied on metallic surfaces, where the first molecular layer is often chemisorbed and thereby thermally stabilized against dewetting and 3D growth. ${ }^{26,27}$ This approach eliminates the requirement of precise thickness-control of the molecular film since any film thickness can be annealed until only the stabilized first molecular layer remains on the inorganic substrate. However, such a thermal stabilization is to date not reported for any TMDC and it is uncertain whether the relatively weak vdW interaction at the OSC-TMDC interface is capable of stabilizing the first molecular layer. Consequently, an in-depth study of the thermal stability of OSCs on TMDCs is of paramount importance, as it serves as a model system for the emerging field of engineering molecular nanostructures ${ }^{28,29}$ and could also enable a scalable fabrication of 2D OSC-TMDC hybrid heterostructures. 
In this work, we present a detailed analysis of the desorption characteristics of ultra-thin pentacene $\left(\mathrm{C}_{22} \mathrm{H}_{14}, \mathrm{PEN}\right)$ and perfluoropentacene $\left(\mathrm{C}_{22} \mathrm{~F}_{14}, \mathrm{PFP}\right)$ films on molybdenum disulfide $\left(\mathrm{MoS}_{2}\right)$. Hybrid systems of PEN and $\mathrm{MoS}_{2}$, in particular, have already been used in various photovoltaic devices and $p-n$ junctions with remarkable properties. ${ }^{16,17,19,21}$ Additional theoretical studies have provided first insight into electronic interactions at the PEN / MoS 2 interface. ${ }^{24,25}$ In contrast to PEN, PFP is an n-type OSC. ${ }^{30}$ Although its carbon backbone is structurally identical to that of PEN, it exhibits a distinctly modified charge distribution due to its electronegative fluorine atoms that lead to an inverted quadrupole moment. Not only does the comparison of these two OSCs enable us to study influences of electronic configuration on the thermal stability of molecular monolayers, but it also provides an interesting prospect for mixed films of PEN and PFP that are additionally stabilized by intermolecular attraction as reported for their multilayer mixtures in a previous work. ${ }^{31}$ To minimize the influence of defects at the $\mathrm{MoS}_{2}$ surface on the OSC-TMDC interface, we use pristine exfoliated $\mathrm{MoS}_{2}$ single crystals on which we found epitaxial growth of PEN and PFP in a previous study. ${ }^{32}$

To study molecular desorption kinetics, we use temperatureprogrammed desorption (TPD) as a versatile technique for investigating the thermal evolution and interfacial bond strength of organic/inorganic hybrid systems. In recent years, TPD has been increasingly employed to study interfaces between large aromatic molecules and metals ${ }^{33-48}$ or graphite. ${ }^{49-52}$ On metals, the activation energy of desorption (often referred to as desorption energy) is commonly used as a measure for the adsorption energy, i.e. the OSC/metal binding energy. On weakly interacting substrates, however, the relation between desorption energy and interface binding energy can be more complex due to an increasing importance of entropy as a result of a larger mobility of the molecular adsorbates. Entropic effects can lead to a thermal stabilization of the first molecular layer in spite of a smaller desorption energy compared to the multilayer, as entropy can reduce the prefactor of desorption, which increases desorption temperatures. ${ }^{50}$ Since prefactors depend on a system's entropy and its partition function, they do not only depend on the molecular species, ${ }^{53}$ but also on the particular interface that can influence molecular mobility. Therefore, prefactors are generally not precisely known, which complicates the determination of reliable desorption energies from TPD as common approximations such as Redhead's method $^{54}$ are not applicable. Moreover, intermolecular interactions can also noticeably influence desorption energies, especially in weakly interacting hybrid systems. While such effects are often mentioned, $36,38,45,47,52$ they are rarely discussed quantitatively and even then, the analysis is restricted to simple linear approximations of coverage-dependent interactions. ${ }^{38,52}$ To study intermolecular interaction energies more accurately and to understand the complex interplay of intermolecular and OSC-TMDC interactions in unitary and mixed monolayers of PEN and PFP, we combine our experimental TPD data with Monte Carlo (MC) simulations that take structural configurations and interactions of molecules into account to compute TPD traces.
In addition to TPD, we use near-edge $X$-ray absorption fine structure (NEXAFS) spectroscopy and work function measurements by means of the Kelvin probe technique to analyze the electronic coupling of the OSCs to $\mathrm{MoS}_{2}$ as well as scanning tunneling microscopy (STM) for a characterization of the geometrical arrangement of the OSC films.

Analysis of our TPD data shows that both PEN and PFP form thermally stabilized monolayers on $\mathrm{MoS}_{2}$ that desorb at significantly higher temperatures than the respective multilayers, although desorption energies are smaller in the monolayer regime than in the multilayer regime. While this, in principle, allows for a preparation of nominal molecular monolayers by means of selective desorption of multilayers, we also find evidence for a significant intermolecular repulsion in unitary PEN and PFP films, which is attributed to their electrostatic interaction. This repulsion, coupled with a relatively weak OSC-2DM interface bond, leads to the formation of a highly mobile, gas-like phase in the unitary molecular (sub) monolayers that prevents the formation of densely packed monolayers at room temperature. However, mutual (electrostatic) attraction of PEN and PFP can be used to increase the packing density in intermixed monolayers, as it favors the formation of ordered co-structures, which is corroborated by STM data.

\section{Methods}

\section{Experimental Details}

PEN (Sigma Aldrich, purity $\geq 99.9 \%$ ) and PFP (Kanto Denka Kogyo, purity $\geq 99 \%$ ) films were grown by means of organic molecular beam deposition under ultrahigh vacuum (UHV) conditions from resistively heated Knudsen cells. If not stated differently, molecular films were grown at a substrate temperature of $270 \mathrm{~K}$ at typical deposition rates of $2 \AA / \mathrm{min}$ as monitored by quartz crystal microbalances.

Similar to a previously described growth protocol, ${ }^{55} \mathrm{MoS}_{2}$ crystals $\left(2 \mathrm{H}-\mathrm{MoS}_{2}\right)$ were grown by means of chemical vapor transport, starting from stoichiometric amounts of Mo, $\mathrm{S}$ and $\mathrm{Br}_{2}$, the latter being the source for the transport agent $\mathrm{MoBr}_{4}$ that is formed in-situ. The reaction was performed in an evacuated quartz glass ampoule that was subjected to a temperature gradient from $1300 \mathrm{~K}$ to $1220 \mathrm{~K}$ for 20 days. This procedure yields crystals of almost centimeter size as shown in reference 32 . The crystals were exfoliated under ambient conditions before being evacuated. Prior to the deposition of molecules, the crystals were annealed at a temperature of $650 \mathrm{~K}$ for $15 \mathrm{~min}$.

TPD measurements were carried out in a dedicated UHV apparatus with a base pressure in the low $10^{-10} \mathrm{mbar}$ regime, as described elsewhere. ${ }^{46,48}$ Briefly, the apparatus hosts a HIDEN EPIC 1000 quadrupole mass spectrometer (QMS) with a mass range up to $1000 \mathrm{amu}$ that enables detection of the intact molecular ions $\operatorname{PEN}^{+}(\mathrm{m} / z=278 \mathrm{amu})$ and $\operatorname{PFP}^{+}(\mathrm{m} / \mathrm{z}=530 \mathrm{amu})$. If not stated differently, TPD traces were recorded with heating rates of $1 \mathrm{k} / \mathrm{s}$. Sample temperatures were precisely measured 
using a calibrated type $\mathrm{K}$ thermocouple mounted on top of the $\mathrm{MoS}_{2}$ crystal.

The microstructural order of molecular thin films was characterized in UHV (base pressure $<10^{-10} \mathrm{mbar}$ ) by means of STM (Omicron VT STM) in constant current mode using etched tungsten tips at sample temperatures of $110 \mathrm{~K}$. The same UHV system hosts a Kelvin Probe (Besocke Delta Phi GmbH, Kelvin Probe $\mathrm{S}$ ) that is used for work function measurements.

NEXAFS measurements in partial electron yield mode (retarding field: $150 \mathrm{~V}$ ) were performed at the HE-SGM dipole beam line of the synchrotron storage ring BESSY II in Berlin (Germany). Details on the experimental setup and the data analysis can be found in reference 56 .

\section{Computational Details}

MC simulations of TPD traces were performed using a modified version of the algorithm described in reference 57 . Molecules are modeled as two-dimensional circular discs to describe an averaged intermolecular interaction and to account for all possible relative rotational orientation that can be expected at elevated temperatures. These discs can diffuse freely on a jellium-like substrate. Intermolecular interactions are modeled by $\mathrm{vdW}$ and Coulomb interactions, the latter leading to a repulsive contribution in unitary films and an attractive contribution in mixed films of PEN and PFP. Structural configurations of the adlayer after molecular diffusion are used as input to compute coverage-dependent desorption energies. Details on the model and the algorithm can be found in the ESIt. The simulated TPD traces that are shown in this work are averaged from at least 50 randomized ensembles. For unitary and mixed PEN and PFP films, we use 64 and 100 molecules, respectively, per calculated TPD trace.

\section{Results and Discussion}

\section{Electronic Coupling and Molecular Orientation}

At first, we performed temperature-dependent NEXAFS measurements of PEN and PFP thin films grown on exfoliated $\mathrm{MoS}_{2}$ single crystals to find out whether the molecular monolayers are thermally stabilized and to obtain information on the molecular orientation at elevated temperatures. Therefore, we deposited $0.5 \mathrm{~nm}$ of the molecules on $\mathrm{MoS}_{2}$ and slowly heated the sample to incrementally increasing temperatures. ${ }^{58}$ The resulting series of spectra are shown in Figures 1 (a) and (b) for PEN and PFP, respectively. Upon annealing of the molecular films, we observe a decrease of the intensity due to desorption of molecules, with residual intensity even after annealing to $410 \mathrm{~K}$. At this temperature, molecular multilayers were found to be completely desorbed on $\mathrm{SiO}_{2}$ and gold, ${ }^{31,41}$ showing that the first molecular layer of PEN and PFP on $\mathrm{MoS}_{2}$ is thermally stabilized relative to the bulk substance. Comparing the monolayer spectra to NEXAFS spectra of multilayers of PEN and PFP on $\mathrm{SiO}_{2}$, where no coupling of the molecules to the substrate occurs (green curves), we identify a modified NEXAFS signature for the PEN monolayer on $\mathrm{MoS}_{2}$ in the sharp $\pi^{*}$-resonances at excitation energies below the $C 1 \mathrm{~s}$

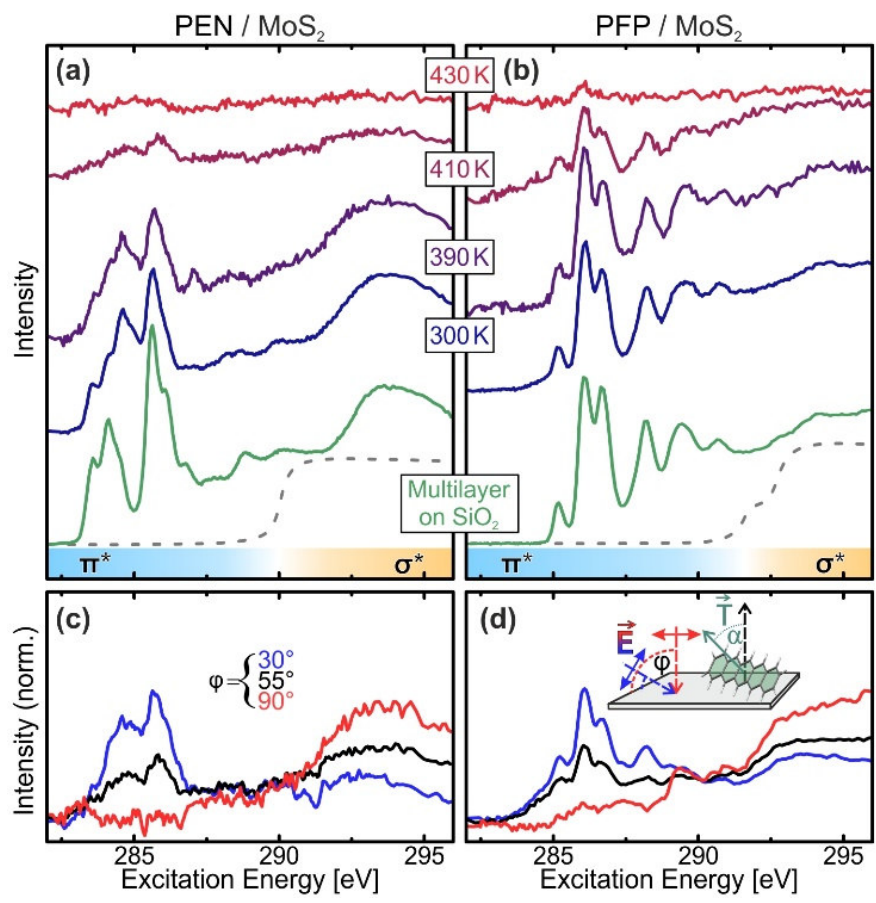

Figure 1 Temperature-dependent $C$ 1s-NEXAFS spectra $\left(\theta=55^{\circ}\right)$ of (a) PEN and (b) PFP films on $\mathrm{MoS}_{2}$ (initial film thickness $0.5 \mathrm{~nm}$; green spectra: multilayers on $\mathrm{SiO}_{2}$ with nominal thickness of $30 \mathrm{~nm}$ recorded at $300 \mathrm{~K}$ ). The dashed lines mark the $\mathrm{C} 1 \mathrm{~s}$ absorption edges. Panels (c) and (d) show C 1s-NEXAFS dichroism measurements of the PEN and PFP films, respectively, after annealing to $410 \mathrm{~K}$.

absorption edge (dashed lines). This is also found for PEN on $\mathrm{Ag}(111)$ and $\mathrm{Au}(111)$ and suggests a small chemical interaction. ${ }^{39,41}$ In contrast, no difference is found for PFP, in line with findings for $\mathrm{Ag}(111) .{ }^{59}$ Complementary Kelvin probe measurements show, however, no changes of the work function of the pristine $\mathrm{MoS}_{2}$ surface upon deposition of unitary monolayers and thin films of both molecular species as well as heterostacks of both species (for details, see the ESIt). Since, in contrast to metals, there is no push-back effect on $\mathrm{MoS}_{2}$ that could compensate work function changes caused by a charge transfer between the molecular films and $\mathrm{MoS}_{2}$, we can conclude that no notable charge transfer between the molecular films and $\mathrm{MoS}_{2}$ takes place. This agrees with a density functional theory study of PEN on $\mathrm{MoS}_{2}{ }^{24}$ and indicates that the electronic coupling of both molecular entities to $\mathrm{MoS}_{2}$ is relatively weak, in spite of the observed modification of the monolayer NEXAFS signature of PEN.

In addition to the thermal stability, we can also use NEXAFS to determine the molecular orientation on the substrate surface by quantitative analysis of the dichroism of NEXAFS spectra recorded at different angles of incidence of the synchrotron radiation as described in detail in reference 56 . In short, since the transition dipole moments (TDMs) corresponding to excitations into unoccupied $\pi^{*}$-orbitals are oriented normal to the molecular backbone (see inset in Figure 1 (d)), the absorption of X-rays with the related photon energies depends on the relative orientation of the molecules and the polarization of the X-ray beam. Thus, one can determine the average orientation of molecules in the film by acquiring NEXAFS spectra at different sample orientations, i.e. at different angles of 
incidence $\varphi$. Figures 1 (c) and (d) show such measurements for acene films that were heated to $410 \mathrm{~K}$. The quantitative analysis of the dichroism of the leading $\pi^{*}$-resonances yields molecular tilt angles of $\alpha<10^{\circ}$, showing that in the (sub-) monolayer regime, PEN and PFP are lying flat on the $\mathrm{MoS}_{2}$ surface even at elevated temperatures.

\section{Thermal Stability of Unitary Monolayers}

To further investigate the nature of the thermally stabilized first layer, TPD measurements with different film thicknesses ranging from $0.5 \AA$ to $12 \AA$ were conducted that are shown in Figures 2 (a) and (b) for PEN and PFP, respectively. For both molecules, two clearly separated peaks are observed. The hightemperature peak occurs at all coverages, but increases in intensity and width only up to nominal film thicknesses of $3 \AA$. From this coverage on, a low-temperature peak begins to form that increases in height with the film thickness but exhibits the same ascending peak flank. As this behavior is characteristic for multilayer desorption, which can be well described by zerothorder desorption kinetics, 60 we ascribe the low-temperature peak to multilayer desorption and the high-temperature peak to monolayer desorption.

In the monolayer regime, we find substantial differences in the desorption traces of the two molecules that range from $380 \mathrm{~K}$ to $490 \mathrm{~K}$ for PEN and from $390 \mathrm{~K}$ to $530 \mathrm{~K}$ for PFP. The higher desorption temperatures of PFP indicate a stronger interaction with $\mathrm{MoS}_{2}$ in comparison to PEN. Since for both acenes, notable multilayer desorption starts at approximately $350 \mathrm{~K}$, the molecular monolayers are sufficiently stabilized to allow for a preparation of nominal monolayers by means of selective desorption of multilayers. This is shown in Figures 2 (c) and (d) for PEN and PFP, respectively, where TPD traces of nominal
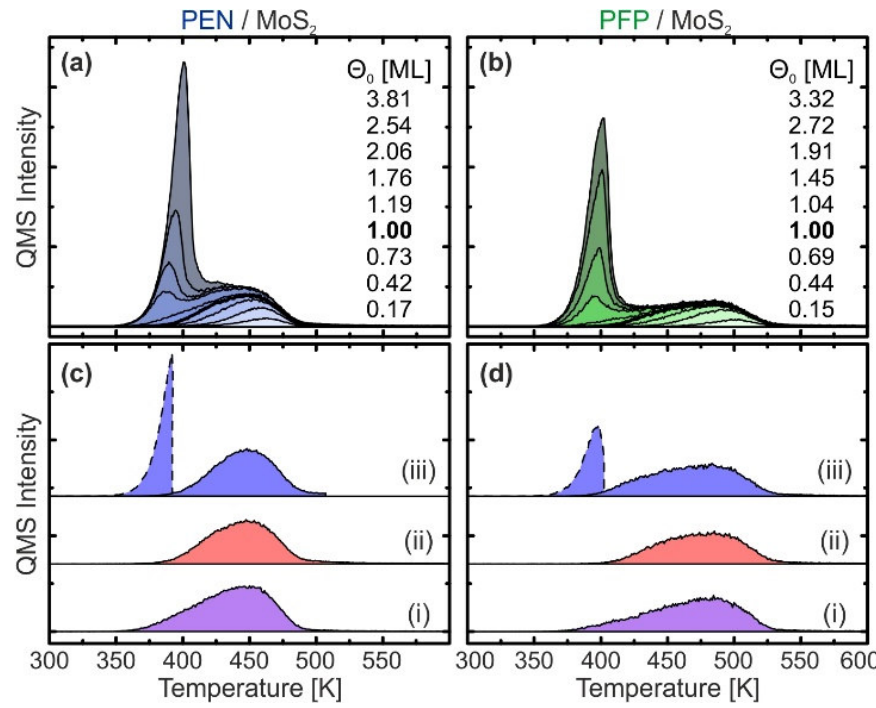

(d)

Figure 2 Series of TPD traces for different film thicknesses of (a) PEN and (b) PFP on $\mathrm{MoS}_{2}$. The nominal monolayers are marked with bold lines. Panels (c) and (d) depict monolayer traces of PEN and PFP, respectively, prepared with three different methods: (i) deposition of $3 \AA$ (nominal monolayer) at $270 \mathrm{~K}$, (ii) deposition of $8 \AA$ at elevated temperature (PEN: $390 \mathrm{~K}$; PFP: $400 \mathrm{~K}$ ) and (iii) deposition of $8 \AA$ at $270 \mathrm{~K}$ and subsequent annealing for $1 \mathrm{~min}$ (PEN: $390 \mathrm{~K}$; PFP: $400 \mathrm{~K}$ ), dashed lines indicate the desorbing multilayers upon annealing. Afterwards, the sample is cooled to $270 \mathrm{~K}$ and then heated again to record the TPD trace (solid line). monolayers prepared by (i) direct deposition of $3 \AA$ of the respective molecule (corresponding to a nominal coverage of $1 \mathrm{ML}$ ) at a substrate temperature of $270 \mathrm{~K}$, (ii) deposition of $8 \AA$ on a crystal at an elevated temperature $(390 \mathrm{~K}$ for PEN and $400 \mathrm{~K}$ for PFP) and (iii) deposition of $8 \AA$ at $270 \mathrm{~K}$ and subsequent annealing at $390 \mathrm{~K}$ (PEN) or $400 \mathrm{~K}$ (PFP) for $1 \mathrm{~min}$ are presented. Both annealing methods yield monolayer TPD traces with no multilayer peak, showing that nominal monolayers of PEN and PFP on $\mathrm{MoS}_{2}$ can be prepared by selective desorption of multilayers. In contrast, method (i) shows a weak low-temperature tail below $400 \mathrm{~K}$ that suggests an onset of multilayer formation, possibly prior to the completion of a densely packed monolayer. Therefore, we use the TPD traces obtained by method (ii), i.e. deposition of $8 \AA$ on a hot $\mathrm{MoS}_{2}$, to define the nominal coverage of $1 \mathrm{ML}$.

The quantitative analysis of TPD traces is based on the PolanyiWigner equation: ${ }^{61}$

$$
r=v \theta^{n} e^{-E_{d} / R T}
$$

Here, $r$ is the desorption rate that is measured in dependence of the substrate temperature $T, \theta$ is the surface coverage, $n$ is the order of desorption kinetics and $R$ is the universal gas constant. The parameters to be determined from the experimental TPD traces are the so-called kinetic parameters, i.e. the activation energy for desorption, or short desorption energy $E_{d}$, and the pre-exponential factor, or prefactor, $v$.

For the quantitative analysis of $E_{d}$ and $v$ in the multilayer regime, we have performed a leading-edge analysis. ${ }^{60}$ This analysis (for details, see the ESI+) yields desorption energies of $(141 \pm 3) \mathrm{kJ} / \mathrm{mol}$ and $(149 \pm 3) \mathrm{kJ} / \mathrm{mol}$ and prefactors of $10^{17.7 \pm 0.4} \mathrm{~s}$ ${ }^{1}$ and $10^{18.5 \pm 0.4} \mathrm{~s}^{-1}$ for PEN and PFP, respectively. These values are in reasonable agreement with the PEN sublimation enthalpy of $(157 \pm 14) \mathrm{kJ} / \mathrm{mol},{ }^{62}$ considering the large margins of error of the literature value. Larger sublimation enthalpies of fluorinated species compared to their non-fluorinated siblings are also reported for naphthalene and octafluoronaphthalene. ${ }^{63}$ This finding can be attributed to a slightly higher polarizability and quadrupole moment as calculated for the case of PEN and PFP, 64 which enable stronger vdW interactions. A comparison of the crystalline phases of PEN and PFP revealed further a somewhat larger Kitaigorodskii packing coefficient for PFP, ${ }^{64}$ which is indicative of stronger dispersion interactions.

In the monolayer regime, the TPD traces do not exhibit the same leading-edge for different initial coverages, which can be seen more clearly in Figures 3 (a) and (b) that depict only the (sub-) monolayer TPD traces of PEN and PFP, respectively. To extract the kinetic parameters from this data, we have used a modified leading-edge analysis ( $m L E A$ ) that is described in detail in a previous publication. ${ }^{48}$ This method uses only small temperature intervals (approximately $20 \mathrm{~K}$ ) at the leading-edge of a TPD trace (corresponding to a coverage change of approx. $1 \%$, details in the Supporting Information) to determine $E_{d}$ and $v$ and can therefore provide coverage-specific values. 

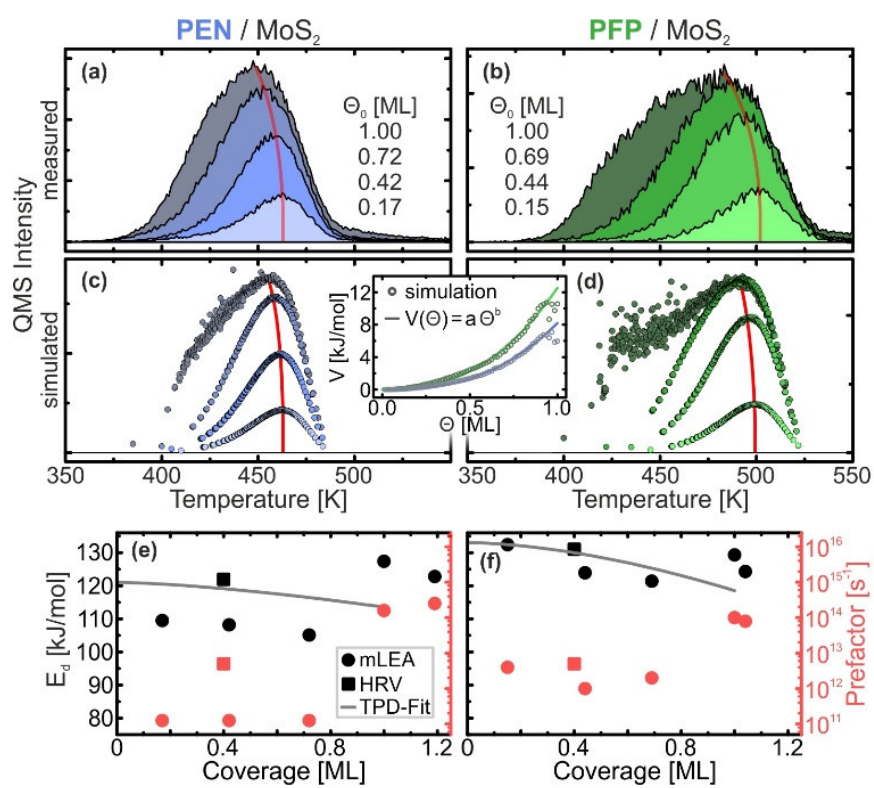

Figure 3 (a) and (b) TPD traces of PEN and PFP, respectively, on $\mathrm{MoS}_{2}$ for different initial (sub-) monolayer coverages $\theta_{0}$. (c) and (d) MC simulations of TPD traces for PEN, respectively, for the same initial coverages as in (a) and (b). The inset shows the intermolecular interaction energy $V$ as a function of the coverage $\theta$ for PEN (blue) and PFP (green) obtained from the MC TPD simulations (circles) with exponential fits (lines). (e) and (f) Coverage-dependent desorption energies $E_{\mathrm{d}}$ (black) and pre-exponential factors $v$ (red) of PEN and PFP, respectively, on $\mathrm{MoS}_{2}$.

The results of the mLEA are presented as circles in Figures 3 (d) and (e) for PEN and PFP, respectively. In the sub-monolayer regime (coverage $<0.8 \mathrm{ML}$ ), we find desorption energies in the range between $105 \mathrm{~kJ} / \mathrm{mol}$ and $110 \mathrm{~kJ} / \mathrm{mol}$ with prefactors of the order of magnitude of $10^{11} \mathrm{~s}^{-1}$ for PEN. Upon saturation of the monolayer, $E_{d}$ increases to $127 \mathrm{~kJ} / \mathrm{mol}$ and $v$ increases to $10^{14} \mathrm{~s}^{-1}$. For PFP, we find larger desorption energies in the range between $121 \mathrm{~kJ} / \mathrm{mol}$ and $132 \mathrm{~kJ} / \mathrm{mol}$ and prefactors of the order of magnitude of $10^{12} \mathrm{~s}^{-1}$ in the submonolayer regime. These values slightly change to $129 \mathrm{~kJ} / \mathrm{mol}$ and $10^{14} \mathrm{~s}^{-1}$, respectively, upon saturation of the monolayer.

The finding of larger desorption energies for the PFP monolayer compared to the PEN monolayer is in line with the observation of an onset of monolayer desorption at higher temperatures. However, all of these kinetic parameters are significantly smaller than the respective results for multi-layer desorption. In particular, desorption energies are significantly smaller in the monolayer than in the multilayer. Since notable multilayer desorption starts at lower temperatures than monolayer desorption, this result is, at first glance, rather surprising and counterintuitive. Notably, the prefactors of both PEN and PFP are smaller than $k_{B} T / h\left(\sim 10^{13} \mathrm{~s}^{-1}\right.$ at $\left.T=400 \mathrm{~K}\right)$, which, according to transition state theory (TST), is the theoretical lowest limit for the prefactor. ${ }^{65}$ This raises the questions whether these results are robust or erroneous, possibly due to the rather low signal-to-noise ratio of the leading edge that is used in the mLEA.

To test the results of the $\mathrm{mLEA}$, we have also performed heating-rate variation (HRV) experiments. Therefore, TPD traces were recorded for a selected coverage at varying heating rates. The heating rate-dependent peak temperature can then be used to derive $E_{d}$ and $v$ independently of each other, as described in detail in reference 48. Because this method requires multiple measurements per initial coverage, only one submonolayer coverage was used per molecule (0.4 ML for PEN and PFP). For precise control of the initial coverages, these submonolayers were prepared by deposition of $8 \AA$ of the respective molecule and subsequent annealing to desorb multilayers and parts of the monolayer. Analysis of the initial coverages from the TPD traces shows that this method yields highly reproducible initial coverages. The HRV data and the corresponding analysis are presented in the ESIt. From the HRV analysis, we obtain $E_{d}=(122 \pm 4) \mathrm{kJ} / \mathrm{mol}$ and $v=10^{12.7 \pm 0.5} \mathrm{~s}^{-1}$ for PEN and $E_{d}=(131 \pm 4) \mathrm{kJ} / \mathrm{mol}$ and $v=10^{12.7 \pm 0.4} \mathrm{~s}^{-1}$ for PFP (squares in Figure 3 ).

Considering the uncertainty of the mLEA (desorption energy error $4 \mathrm{~kJ} / \mathrm{mol}$ and $9 \mathrm{~kJ} / \mathrm{mol}$ and prefactor error 0.5 and 1.1 orders of magnitude for PEN and PFP, respectively), these HRV results are in fair agreement with those of the mLEA and thus confirm the general trend. Although the HRV desorption energies are slightly larger than those obtained from the mLEA, they are still significantly smaller than those of the multilayers. The HRV prefactors are an order of magnitude larger than those obtained from the mLEA and thus in agreement with the lower limit from TST, but still orders of magnitude smaller than typical prefactors obtained in TPD experiments with large molecules. ${ }^{34,49,50,53,65-70}$

This effect, i.e. a small desorption energy in the monolayer compared to the multilayer in combination with an increase of the prefactor upon saturation of the monolayer by several orders of magnitude, has already been observed and described in detail for 2,4'-bis(terpyridine) on highly oriented pyrolytic graphite (HOPG). 50 An explanation for this phenomenon is provided by TST: The reaction rate constant $k_{\mathrm{TST}}$ for the transition from the adsorbed initial state (IS) to the transition state (TS), that is the rate of desorption, can be related to the standard Gibbs free energy of activation, $\Delta G^{\ddagger}$ (for a more detailed derivation, see the $\mathrm{ESI}+)^{: 71}$

$$
k_{\mathrm{TST}}=\frac{k_{\mathrm{B}} T}{h} e^{-\Delta G^{\ddagger} / R T} .
$$

Using equation 2 and the relation $\Delta G^{\ddagger}=\Delta H^{\ddagger}-T \Delta S^{\ddagger}$, where $\Delta H^{\ddagger}$ is the standard enthalpy of activation and $\Delta S^{\ddagger}$ is the standard entropy of activation, one can identify the desorption energy as ${ }^{71}$

$$
E_{d}=\Delta H^{\ddagger}+R T
$$

With equation 3 , equation 2 can be brought into the form $k_{\mathrm{TST}}=v \mathrm{e}^{-E_{d} / R T}$, which allows to identify the prefactor as

$$
v=\frac{k_{B} T}{h} \mathrm{e}^{\left(\Delta S^{\ddagger} / R\right)+1} \text {. }
$$

On this basis, the exceptionally small prefactors can be interpreted. Equation 4 shows that the prefactor scales with the entropy gain upon transition from the adsorbed IS to the TS. 
When a chemisorbed molecule reaches the TS for desorption, it typically gains additional degrees of freedom (DOFs) compared to the IS, because bonds to the surface break and formerly frustrated translations and rotations turn into (nearly) free translations and rotations. Therefore, the partition function in the transition state is frequently larger than in the adsorbed state, giving rise to a desorption prefactor larger than $k_{B} T / h .^{72}$

If the prefactor is in the range of $k_{B} T / h$ or even smaller, as is the case here, then the situation must be reversed, i.e. the TS must have a reduced number of DOFs, compared to the IS. This is the case when the adsorbed molecules are very mobile, while certain geometry restrictions apply to the TS. According to STM (see below), the adsorbed PEN molecules are indeed very mobile, resulting in large partition functions for two translation DOFs and one rotational DOF (around the axis perpendicular to the surface). In addition, due to the weak interaction with the surface, the two remaining frustrated rotations and one remaining frustrated translation (vibration perpendicular to the surface) are expected to contribute substantially to the total partition function. Especially the latter contributes only to the IS and can therefore substantially reduce the desorption prefactor.

Regarding the geometry of the TS, it is obvious that the potential energy of a large planar molecule above a surface depends not only on the distance between its center-of-mass and the surface, but also on its orientation relative to the surface. This implies that the lowest-energy path of the desorption process is closely related to geometric parameters. Hence, of the many adsorption configurations available for the mobile adsorbed molecule, not all are equally suitable for the TS. The reduced number of configurations in the TS means that the partition function is reduced compared to the IS. In addition, a large planar molecule such as pentacene needs to be rather far away from the surface before it can freely rotate around all three axes. Geometry considerations for the case of pentacene show that its center-of-mass must be at least $\sim 7 \AA$ above the surface, which far exceeds the vdW distance of $<3 \AA$ between the molecular plane and the substrate..$^{73}$ It is therefore possible that the rotational partition function in the TS is still reduced compared to that of the gas phase. In contrast to the mobile molecules in the (sub-) monolayers, multilayers of PEN and PFP form crystalline films, as shown in a previous study. ${ }^{32}$ In these films, the molecules are immobile, resulting in a larger entropy gain upon desorption and therefore a larger prefactor for desorption.

An energetic argument for the stabilization of the mobile molecular monolayer can be made on the basis of equation 2, which illustrates that the relevant thermodynamic potential for desorption is the standard Gibbs free energy of activation, not the standard enthalpy of activation that is closely related to the desorption energy. Consequently, the energy barrier for desorption does not only depend on the desorption energy, but also on the entropy change and thus on the prefactor. Using equations 3 and 4 , one can calculate $\Delta G^{\ddagger}{ }_{\text {mono }}-\Delta G^{\ddagger}$ multi , i.e. the difference in the standard Gibbs free energies of activation for desorption from mono- and multilayer (for details, see the ESIt):
$\Delta G_{\text {mono }}^{\ddagger}-\Delta G_{\text {multi }}^{\ddagger}=E_{d, \text { mono }}-E_{d, \text { multi }}+R T \ln \left(\frac{\nu_{\text {multi }}}{v_{\text {mono }}}\right)$

At $400 \mathrm{~K}$, equation 5 yields a difference of the mono- and multilayer standard Gibbs free energy of activation of $19 \mathrm{~kJ} / \mathrm{mol}$ for PEN and $26 \mathrm{~kJ} / \mathrm{mol}$ for PFP, using the results of the HRV for the monolayer parameters. This shows that for both molecules, the barrier for desorption, given by the standard Gibbs free energy of activation, is larger in the monolayer than in the multilayer, which explains the stabilization of the molecular monolayers. The stabilization of the PEN and PFP monolayers is thus caused by entropy due to the formation of a highly mobile gas phase rather than a strong interface bond.

Upon saturation of the monolayer, the results of the mLEA (squares in Figures $3(e)$ and (f)) show that the prefactors of PEN and PFP increase to about $10^{14} \mathrm{~s}^{-1}$, indicating a reduction of the molecular mobility. Comparing the prefactors for monolayer desorption to those for the multilayers, they are, however, still several orders of magnitude smaller. This indicates that only multilayer growth can reduce molecular mobility, which allows to conclude that no close-packed and therefore well-ordered monolayers of PEN and PFP are formed on $\mathrm{MoS}_{2}$ at room temperature, as observed for PFP on $\mathrm{Ag}(111)$ in a previous study. ${ }^{59}$ Hence, the nominal monolayer that can be prepared by selective desorption of multilayers does not correspond to a densely and therefore complete monolayer.

\section{Intermolecular Repulsion}

A microscopic explanation for the formation of a highly mobile gas phase in the molecular monolayers can be found upon closer inspection of the (sub-) monolayer TPD traces. Figures 3 (a) and (b) show only the monolayer TPD traces of PEN and PFP, respectively, that exhibit a distinct peak shift to lower temperatures for increasing coverages as indicated by red lines. Such a peak shift is already reported for other systems including PEN on $\mathrm{Au}(111)$ and commonly attributed to (sometimes substrate-mediated) intermolecular repulsion. ${ }^{36,38,45,47,52}$ This causes a reduction of the desorption energy for increasing coverages as the repulsion increases with decreasing average nearest-neighbor distances. Such a reduction of the desorption energy at increasing coverage can also be found in Figures 3 (e) and (f) in the mLEA results for both molecules.

Using MC TPD simulations with electrostatic intermolecular interactions that are based on the molecular quadrupole moments calculated by density functional theory, ${ }^{64}$ we can find an estimate for the intermolecular interaction energies. Starting with the charge distribution of isolated molecules that exhibit a distinct quadrupole moment, the molecular charge distributions were represented by discrete point charges, which mediate the lateral electrostatic coupling. Slight adjustment of the effective charges in our disc shaped molecules allows to reproduce the experimentally observed coverage-dependence of the TPD traces. The resulting simulated TPD traces are presented in Figures 3 (c) and (d) for PEN and PFP, respectively. With kinetic parameters that are close to those derived from the HRV experiments (PEN: $E_{d}=121 \mathrm{~kJ} / \mathrm{mol}, v=10^{12.5} \mathrm{~s}^{-1}$; PFP: 
$\left.E_{d}=133 \mathrm{~kJ} / \mathrm{mol}, v=10^{12.7} \mathrm{~s}^{-1}\right)$, the lowest coverage TPD traces can be reproduced accurately with Coulomb charges of $q=$ 0.07 e for PEN and $q=0.105$ e for PFP (for details on the modeled charge distribution, see the ESIt). The Coulombic intermolecular repulsion leads, as expected, to a peak shift towards lower temperatures for increasing coverages as well as a significant broadening of the TPD traces of the saturated monolayers, in particular in the case of PFP. Only at intermediate coverages, the simulated coverage-dependent peak shift is less pronounced than the experimentally observed shift. This suggests that the real interaction potential has a somewhat longer range, i.e. decreases less strongly with distance, than the one used for the simulation.

Our simulation further allows to derive an effective potential $V$ for intermolecular interactions as a function of the surface coverage $\theta$ that is inversely related to the average intermolecular nearest-neighbor distance. The effective lateral interaction potentials of PEN and PFP obtained from our MD simulations are shown as circles in the inset of Figures 3 (c) and (d). Both curves can be modeled accurately by a simple exponential function of the form $V(\theta)=a \theta^{b}$. Fitting of this function to the simulated potential yields similar exponents $b$ for PEN and PFP with $(2.6 \pm 0.1)$ and $(2.5 \pm 0.1)$, respectively. For the maximum interaction energy that is given by the factor $a$, we find $(8.2 \pm 0.1) \mathrm{kJ} / \mathrm{mol}$ and $(12.5 \pm 0.2) \mathrm{kJ} / \mathrm{mol}$ for PEN and PFP, respectively. This corresponds to $\sim 7 \%$ and $\sim 9 \%$ of the desorption energy in the zero-coverage limit, respectively, in line with a result of $\sim 7 \%$ reported for para-hexaphenyl on $\mathrm{Au}(111)$. $^{38}$

The coverage-dependence of the interaction potential obtained from our MC TPD simulation can be plugged into the PolanyiWigner equation (equation 1 ) to determine the interaction energies directly from the experimental data. Therefore, we have used the prefactors that were also used for the MC TPD simulations, which are in agreement with the HRV results, and fitted the Polanyi-Wigner equation with a coverage-dependent desorption energy $E_{d}(\theta)=E_{0}-a \theta^{b}$ to the sets of TPD traces shown in Figures $3(a)$ and (b). The resulting fits (see the ESI+) are in excellent agreement with the experimental TPD traces for all coverages. For the desorption energies (lines in Figures 3 (e) and $(\mathrm{f}))$, we find $E_{d}(\theta)=\left(121-7.5 \theta^{1.5}\right) \mathrm{kJ} / \mathrm{mol}$ for PEN and $E_{d}(\theta)=\left(133-14.5 \theta^{1.7}\right) \mathrm{kJ} / \mathrm{mol}$ for PFP. In comparison to the simulation, the exponents are significantly smaller, reflecting the finding that our model somewhat underestimates the range of the intermolecular interactions. Nonetheless, the maximum interaction energies are of the same order of magnitude as those of the simulation with $7.5 \mathrm{~kJ} / \mathrm{mol}$ for PEN and $14.5 \mathrm{~kJ} / \mathrm{mol}$ for PFP and therefore still of the order of magnitude of only $10 \%$ of the zero-coverage desorption energy.

\section{Intermixed Monolayers}

The above discussed TPD data shows that the unitary submonolayer phases of PEN and PFP are mainly entropically stabilized, i.e. by an entropy gain through the high mobility of the adsorbed molecules. These are unfavorable conditions for the formation of stable, densely packed monolayers, in which the mobility of the molecules and thus the entropic stabilization of the layer would be reduced. Therefore, it is desirable to achieve enthalpic stabilization of the monolayer by introducing attractive intermolecular interactions, which result in the formation of ordered, close-packed molecular monolayers.

In a previous study on intermixed thin multilayer films of PEN and PFP, we found evidence for intermolecular attraction. ${ }^{31}$ To test whether these attractive forces also occur laterally in monolayer films with a flat-lying molecular orientation, we have conducted further TPD measurements on mixed monolayers of
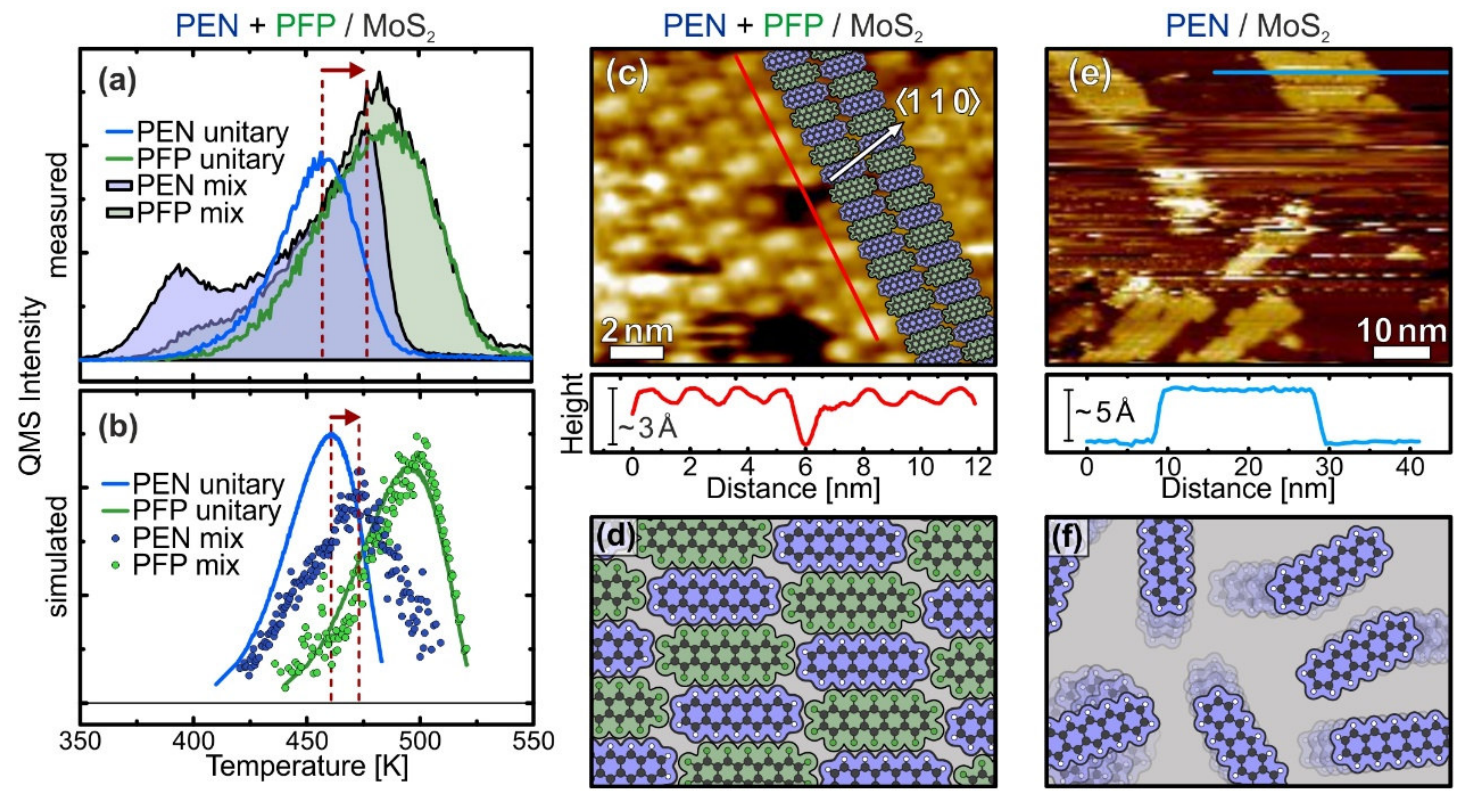

Figure 4 (a) Quasi-simultaneously recorded TPD traces of 0.6 ML PEN and 0.8 ML PFP in a mixed film (filled traces) and unitary films of 0.4 ML PEN (blue line) and 0.7 ML PFP (green line) on $\mathrm{MoS}_{2}$. (b) Simulated TPD traces of a randomized mixture of $0.5 \mathrm{ML}$ PEN and 0.5 ML PFP (dots), $0.5 \mathrm{ML}$ PEN (blue line) and 0.5 ML PFP (green line). (c) STM micrograph $(-2.5 \mathrm{~V}, 250 \mathrm{pA}, 110 \mathrm{~K})$ of an intermixed monolayer of PEN and PFP on $\mathrm{MoS}_{2}$ with a linescan. The inset illustrates the alternating molecular structure. The substrate azimuth was determined from low-energy electron diffraction. (d) Illustration of the intermixed monolayer. (e) STM micrograph (-3.76 V, 120 pA, $110 \mathrm{~K})$ of $8 \AA$ PEN on MoS 2 after annealing to $390 \mathrm{~K}$ for $1 \mathrm{~min}$. (f) Illustration of a highly mobile (sub-) monolayer of PEN. 
PEN and PFP. Figure 4 (a) shows the quasi-simultaneously recorded TPD traces of PEN (filled blue curve) and PFP (filled green curve) from an equimolar intermixture with a coverage slightly exceeding $1 \mathrm{ML}$, resulting in small multilayer desorption peaks. In comparison to the unitary PEN monolayer (blue line), the ascending flank of the PEN monolayer signal of the mixed film (filled blue curve) is less steep and the peak maximum shifts by approximately $20 \mathrm{~K}$ to higher temperatures, followed by a rather sharp high-temperature tail. In contrast, the TPD trace of PFP (filled green curve) is almost identical to the unitary phase (green line). Above $500 \mathrm{~K}$, the TPD traces of unitary and intermixed PFP are equal because PEN has completely desorbed, leaving a nearly pristine PFP film on the surface.

Due to a continuous variation of the stoichiometric ratio of PEN:PFP during the desorption experiment, the desorption parameters are continuously changing. This is equivalent to a superposition of many partially overlapping desorption peaks that are slightly shifted on the temperature axis with respect to each other. This results in a distorted leading-edge that yields unreasonable results using the mLEA. A HRV analysis is difficult since precise reproducibility of two coverages (for PEN and PFP) is needed to achieve identical samples for the several required experiments with different heating rates. Hence, the TPD traces of the mixed film were only analyzed qualitatively. The modified desorption behavior compared to the unitary phases of PEN and PFP can be explained by an interplay of intermolecular and molecule-substrate interactions. From our TPD traces of unitary PEN and PFP monolayers, we know that PFP desorbs at higher temperatures than PEN. Due to an electrostatic attraction between the opposing quadrupole moments of PEN and PFP, 64 PFP acts as an anchor for PEN by increasing its desorption energy. However, since PFP binds stronger to $\mathrm{MoS}_{2}$ than PEN, PEN still desorbs at a higher rate than PFP until finally, only PFP remains on the substrate surface. At this point, the desorption rate of PEN rapidly drops to zero and the descending flanks of the unitary and intermixed phase PFP monolayer TPD traces are equal.

This behavior is well reproduced by our MC TPD simulations, as shown in Figure 4 (b). Note that the same interaction parameters were used for the simulation of TPD traces of unitary and mixed films of PEN and PFP. As in the experiment, we find a shift of the PEN desorption peak of the mixed film (blue dots) to higher temperatures, i.e. closer to the PFP peak, in comparison to the unitary PEN film (blue line). Although the simulated peak shift is somewhat smaller with only approximately $15 \mathrm{~K}$ in comparison to the experiment, the slope of the ascending flank of PEN in the mixed film is reduced in comparison to the unitary film, as in the experiment. For PFP, the simulation shows no significant difference between unitary (green line) and mixed phase (green dots), which agrees well with the experiment. Only a slight shift of the PFP peak of the mixed film to higher temperatures can be seen that indicates a stabilization of PFP beyond the stabilization of the unitary PFP monolayer due to the mutual attraction of PEN and PFP.

The above discussed TPD data illustrates the importance of intermolecular interactions for the kinetic parameters, showing that molecular monolayers can be stabilized by electrostatic attraction in mixed films. However, since a quantitative analysis of the TPD data is not possible at the current state for mixed films of PEN and PFP as the mixing ratio of PEN and PFP changes dynamically, no conclusion can be drawn on the molecular mobility in the mixed film. To find out whether the mutual attraction of PEN and PFP can reduce molecular mobility and lead to the formation of an ordered, densely packed molecular layer, we have performed STM measurements on unitary and mixed films at temperatures of $110 \mathrm{~K}$. Figure 4 (c) shows an STM micrograph of a mixed monolayer of PEN and PFP that clearly shows an ordered molecular structure. A contrast between neighboring molecules is visible that is caused by different electronic properties of PEN and PFP, showing that a wellordered stoichiometric 1:1 intermixture is formed as illustrated in the inset of the figure. The linescan along a molecular row (bottom panel) shows the alternating structure of the mixed film more clearly. A vacancy in the molecular adlayer reveals a layer height of approximately $3 \AA$, corresponding to a flat-lying molecular orientation. A more detailed analysis of the molecular geometry can be found in the Supporting Information. The molecular arrangement is illustrated in Figure 4 (d).

In addition to the mixed phase, we have also conducted STM measurements on unitary monolayers on $\mathrm{MoS}_{2}$. Imaging of these films has proven to be rather challenging, which can be directly attributed to the high lateral mobility of the molecules. Figure 4 (e) shows an STM micrograph of $4 \AA$ PEN on $\mathrm{MoS}_{2}$, corresponding to a coverage slightly above one monolayer. While no molecular structure could be resolved in the first layer and the image shows areas of instable contact caused by frequent tip changes, it is possible to image molecular islands in the second layer. A linescan across a bilayer island (bottom panel) reveals a step height of about $5 \AA$, which is larger than the typical height of $3 \AA$ observed for flat-lying acenes. These findings are perfectly in line with our NEXAFS and TPD data: In the (sub-) monolayer regime, PEN lies flat on the $\mathrm{MoS}_{2}$ surface. Due to their mobility, it is not possible to image molecules in the first layer at a temperature of $110 \mathrm{~K}$, as illustrated in Figure 4 (f). Upon multilayer growth, however, PEN tilts and forms a herringbone structure, leading to condensation of the highly mobile phase and formation of islands that can be imaged by STM. The molecular tilt in the multilayer phase causes an increased step height between the flat-lying first and tilted second on tilted first layer, as reported for PEN on HOPG in a previous study. ${ }^{43}$

The combination of STM with TPD reveals that the mutual electrostatic attraction of PEN and PFP is indeed capable of inducing the formation of well-ordered, close-packed molecular monolayers on $\mathrm{MoS}_{2}$, at least at a temperature of $110 \mathrm{~K}$, at which PEN is still mobile in its unitary phase. In the mixed film, the attractive forces between PEN and PFP add an enthalpic stabilization, which compensates for a potential loss of entropic stabilization. 


\section{Conclusions}

In this study, we show that PEN and PFP on $\mathrm{MoS}_{2}$ form (sub-) monolayers that are stabilized with respect to their multilayer phases, preventing dewetting and 3D growth of the nominal monolayer. In contrast to, for instance, adsorption on metallic surfaces, the origin of this stabilization lies in the entropy of the mobile molecular film rather than the strength of the interfacial bond. This entropic stabilization effect has previously been reported for 2,4'-bis(terpyridine) on HOPG, 50 another planar polycyclic aromatic hydrocarbon on a weakly interacting substrate and can be expected to be a general phenomenon occurring for planar polycyclic aromatic molecules adsorbed on weakly interacting substrates (with a small corrugation of the molecule-substrate interaction potential).

The high mobility of the molecules in the unitary monolayers can be ascribed to a combination of a relatively weak interface bond and intermolecular repulsion. Consequently, the entropic stabilization is an interface effect that can only occur in the (sub) monolayer regime, as is illustrated in Figure 5: In multilayer films, molecules adopt packing motifs that lead to stabilizing attractive contributions to the intermolecular interactions, for instance a herringbone packing motif (Figure $5(a)$ ), whereas in (sub-) monolayers, molecules are forced into a flat-lying orientation by the substrate that results in a repulsive intermolecular interactions in unitary films (Figure 5 (b)) and attractive interactions between PEN and PFP (Figure 5 (c)).

These findings show that, in spite of a relatively weak interface bond, nominal molecular monolayers can be prepared on TMDCs and other weakly interacting substrates by means of selective desorption of multilayers, which is a more scalable process than the direct deposition of monolayers. The stabilization comes, however, at the price of reduced structural order and packing density: a mobile monolayer cannot be a highly ordered nanostructure. Nonetheless, such monolayers can find technical applications, for instance as spacer layers between layers of 2DMs.

Finally, we have shown that attractive intermolecular interactions can further stabilize molecular monolayers even though attraction is likely to reduce the entropic stabilization of unitary films, thus paving the way for the preparation of highly ordered molecular films on weakly interacting substrates. Attractive intermolecular interactions in monolayers are often found in heterostructures of fluorinated and non-fluorinated OSCs, ${ }^{74-78}$ so the concept of stabilization by intermolecular (a)

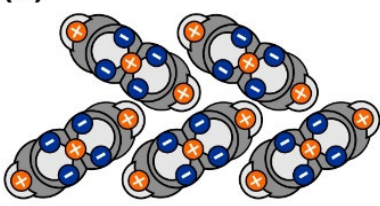

(b)

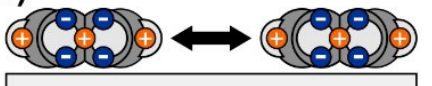

(c)

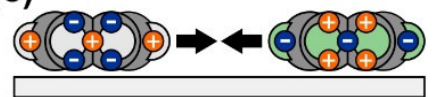

Figure 5 Illustration of electrostatic intermolecular interactions of PEN in a multilayer film with a herringbone packing motif (a), in a (sub-) monolayer film of PEN (b) and a mixed (sub-) monolayer film of PEN and PFP (c), shown in a side view along the long molecular axis. The molecular quadrupole moments are indicated by negative (blue) and positive (orange) point charges. attraction should be applicable beyond our model system of PEN and PFP. Molecules that are only partially fluorinated, such as the unilaterally fluorinated 1,2,10,11,12,14hexafluoropentacene, ${ }^{79}$ could extend this concept to homomolecular films.

\section{Author contributions}

G.W. and T.B. initiated the project. T.B: and P.-M.D. performed the NEXAFS measurements. P.-M.D. performed the STM and Kelvin probe measurements and developed and ran the MC TPD simulations. S.R.K., P.-M.D. and T.B. performed the TPD measurements. S.R.K. analyzed the experimental TPD data. P.M.D., G.W., S.R.K. and J.M.G. wrote the manuscript.

\section{Conflicts of interest}

There are no conflicts to declare.

\section{Acknowledgements}

We gratefully acknowledge financial support from the German Research Foundation (DFG) in the framework of the Collaborative Research Center 'Structure and Dynamics of Internal Interfaces' (223848855-SFB 1083, TP A2 and TP A4) and the Helmholtz-Zentrum Berlin (electron storage ring BESSY II) for provision of synchrotron radiation at the HE-SGM beamline. We further thank Steffen Zörb (Fachbereich Chemie, PhilippsUniversität Marburg) for providing $\mathrm{MoS}_{2}$ crystals.

\section{Notes and references}

1 K. S. Novoselov, A. K. Geim, S. V. Morozov, D. Jiang, Y. Zhang, S. V. Dubonos, I. V. Grigorieva and A. A. Firsov, Science, 2004, 306, 666-669.

2 K. S. Novoselov, A. Mishchenko, A. Carvalho and A. H. Castro Neto, Science, 2016, 353, aac9439.

3 Q. H. Wang, K. Kalantar-Zadeh, A. Kis, J. N. Coleman and M. S. Strano, Nat. Nanotechnol., 2012, 7, 699-712.

4 L. Britnell, R. V. Gorbachev, R. Jalil, B. D. Belle, F. Schedin, A. Mishchenko, T. Georgiou, M. I. Katsnelson, L. Eaves, S. V. Morozov, N. M. R. Peres, J. Leist, A. K. Geim, K. S. Novoselov and L. A. Ponomarenko, Science, 2012, 335, 947-950.

5 L. Britnell, R. M. Ribeiro, A. Eckmann, R. Jalil, B. D. Belle, A. Mishchenko, Y.-J. Kim, R. V. Gorbachev, T. Georgiou, S. V. Morozov, A. N. Grigorenko, A. K. Geim, C. Caisraghi, A. H. Castro Neto and K. S. Novoselov, Science, 2013, 340, 13111314.

6 A. K. Geim and I. V. Grigorieva, Nature, 2013, 499, 419-425.

7 F. Withers, O. Del Pozo-Zamudio, S. Schwarz, S. Dufferwiel, P. M. Walker, T. Godde, A. P. Rooney, A. Gholinia, C. R. Woods, P. Blake, S. J. Haigh, K. Watanabe, T. Taniguchi, I. L. Aleiner, A. K. Geim, V. I. Fal'ko, A. I. Tartakovskii and K. S. Novoselov, ACS Nano Lett., 2015, 15, 8223-8228.

8 M. Q. Zeng, Y. Xiao, J. X. Liu, K. Yang and L. Fu, Chem. Rev., 2018, 118, 6236-6296.

9 D. Akinwande, N. Petrone and J. Hone, Nat. Commun., 2014, 5, 5678.

10 W. Zhu, S. Park, M. N. Yogeesh and D. Akinwande, Flex. Print. Electron., 2017, 2, 043001. 
11 M. Gobbi, E. Orgiu and P. Samori, Adv. Mater., 2018, 30, 1706103.

12 Y. L. Huang, Y. J. Zheng, Z. Song, D. Chi, A. T. S. Wee and S. Y. Quek, Chem. Soc. Rev., 2018, 47, 3241-3264.

13 Y. Huang, W. Zheng, Y. Qiu and P. Hu, ACS Appl. Mater. Interfaces, 2016, 8, 23362-23370.

14 L.-Y. Gan, Q. Zhang, Y. Cheng and U. Schwingenschlögl, J. Phys. Chem. Lett., 2014, 5, 1445-1449.

15 S. Vélez, D. Ciudad, J. Island, M. Buscema, O. Txoperena, S. Parui, G. A. Steele, F. Casanova, H. S. J. van der Zant, A. Castellanos-Gomez and L. E. Hueso, Nanoscale, 2015, 7, 15442-15449.

16 D. Jariwala, S. L. Howell, K.-S. Chen, J. Kang, V. K. Sangwan, S. A. Filippone, R. Turrisi, T. J. Marks, L. J. Lauhon and M. C. Hersam, ACS Nano Lett., 2016, 16, 497-503.

17 S. B. Homan, V. K. Sangwan, I. Balla, H. Bergeron, E. A. Weiss and M. C. Hersam, ACS Nano Lett., 2017, 17, 164-169.

18 R. Chen, C. Lin, H. Yu, Y. Tang, C. Song, L. Yuwen, H. Li, X. Xie, L. Wang and W. Huang, Chem. Mater., 2016, 28, 4300-4306.

19 J.-K. Kim, K. Cho, T.-Y. Kim, J. Pak, J. Jang, Y. Song, Y. Kim, B. Y. Choi, S. Chung, W.-K. Hong and T. Lee, Sci. Rep., 2016, 6, 36775.

20 C. E. Petoukhoff, M. B. M. Krishna, D. Vori, I. Bozkurt, S. Deckoff-Jones, M. Chhowalla, D. M. O'Carroll and K. M. Dani, Phys. Rev. Lett., 2002, 89, 196103.

21 J. Dong, F. Liu, F. Wang, J. Wang, M. Li, Y. Wen, L. Wang, G. Wang, J. He and C. Jiang, Nanoscale, 2017, 9, 7519-7525.

22 H. J. Park, C.-J. Park, J. Y. Kim, M. S. Kim, J. Kim and J. Joo, ACS Appl. Mater. Interfaces, 2018, 10, 32556-32566.

23 L. Zhang, A. Sharma, Y. Zhu, Y. Zhang, B. Wang, M. Dong, H. T. Nguyen, Z. Wang, B. Wen, Y. Cao, B. Liu, X. Sun, J. Yang, Z. Li, A. Kar, Y. Shi, D. Macdonald, Z. Yu, X. Wang and Y. Lu, Adv. Mater., 2018, 30, 1803986.

24 N. Shen and G. Tao, Adv. Mater. Interfaces, 2017, 4, 1601083.

25 X.-Y. Xie, X.-Y. Liu, Q. Fang, W.-H. Fang and G. Cui, J. Phys. Chem. A, 2019, 123, 7693-7703.

26 S. Söhnchen, S. Lukas and G. Witte, J. Chem. Phys., 2004, 121, 525-534.

27 F. Widdascheck, A. A. Hauke and G. Witte, Adv. Funct. Mater., 2019, 1808385.

28 J. V. Barth, G. Costantini and K. Kern, Nature, 2005, 437, 671679.

29 H. Liu, J. Xu, Y. Li and Y. Li, Acc. Chem. Res., 2010, 43, 14961508.

30 Y. Sakamoto, T. Suzuki, M. Kobayashi, Y. Gao, Y. Fukai, Y. Inoue, S. Fumio and S. Tokito, J. Am. Chem. Soc., 2004, 126, 8138-8140.

31 T. Breuer and G. Witte, J. Chem. Phys., 2013, 138, 114901.

32 T. Breuer, T. Maßmeyer, A. Mänz, S. Zoerb, B. Harbrecht and G. Witte, Phys. Status Solidi RRL, 2016, 10, 905-910.

33 S. Lukas, S. Vollmer, G. Witte and C. Wöll, J. Chem. Phys., 2001, 114, 10123-10130.

34 K. A. Fichthorn and R. A. Miron, Phys. Rev. Lett., 2002, 89, 196103.

35 S. Müllegger, I. Salzmann, R. Resel, G. Hlawacek, C. Teichert and A. Winkler, J. Chem. Phys., 2004, 121, 2272-2277.

36 S. Müllegger, O. Stranik, E. Zojer and A. Winkler, Appl. Surf. Sci., 2004, 221, 184-196.

37 S. Müllegger and A. Winkler, Surf. Sci., 2005, 574, 322-330.

38 S. Müllegger and A. Winkler, Surf. Sci., 2006, 600, 1290-1299.

39 D. Käfer and G. Witte. Chem. Phys. Lett., 2007, 442, 376-383.

40 P. Frank, N. Koch, M. Koini, R. Rieger, K. Müllen, R. Resel and A. Winkler, Chem. Phys. Lett., 2009, 473, 321-325.

41 D. Käfer, C. Wöll and G. Witte, Appl. Phys. A, 2009, 95, 273284.

42 P. Frank, T. Djuric, M. Koini, I. Salzmann, R. Rieger, K. Müllen, R. Resel, N. Koch and A. Winkler, J. Phys. Chem. C, 2010, 114, 6650-6657.
43 J. Götzen, D. Käfer and G. Witte, Phys. Rev. B, 2010, 81, 085440.

44 A. Winkler, Surf. Sci., 2016, 643, 124-137.

45 F. Maaß, Y. Jiang, W. Liu, A. Tkatchenko and P. Tegeder, J. Chem. Phys., 2018, 148, 214703.

46 B. P. Klein, N. van der Heijden, S. R. Kachel, M. Franke, C. K. Krug, K. K. Greulich, L. Ruppenthal, P. Müller, P. Rosenow, S. Parhizkar, F. C. Bocquet, M. Schmid, W. Hieringer, R. J. Maurer, R. Tonner, C. Kumpf, I. Swart and J. M. Gottfried, Phys. Rev. X, 2019, 9, 011030.

47 F. Maaß, M. Ajdari, F. C. Kabeer, M. Vogtland, A. Tkatchenko and P. Tegeder, J. Phys. Chem. Lett., 2019, 10, 1000-1004.

48 S. R. Kachel, B. P. Klein, J. M. Morbec, M. Schöninger, M. Hutter, M. Schmid, P. Kratzer, B. Meyer, R. Tonner and J. M. Gottfried, J. Phys. Chem. C, 2020, 124, 8257-8268.

49 R. Zacharia, H. Ulbricht and T. Hertel, Phys. Rev. B, 2004, 69, 155406.

50 M. Roos, A. Breitruck, H. E. Hoster and R. J. Behm, Phys. Chem. Chem. Phys., 2010, 12, 818-822.

51 J. Weippert, J. Hauns, J. Bachmann, A. Böttcher, X. Yao, B. Yang, A. Narita, K. Müllen and M. M. Kappes, J. Chem. Phys., 2018, 149, 194701.

52 J. Weippert, P. Huber, A. Schulz, K. Y. Amsharov, A. Böttcher and M. M. Kappes, Phys. Status Solidi RRL, 2019, 1900348.

53 S. L. Tait, Z. Dohnálek, C. T. Campbell and B. D. Kay, J. Chem. Phys., 2005, 122, 164708.

54 P. A. Redhead, Vacuum, 1962, 12, 203-211.

55 C. Piña, P. Bosch, D. Acosta, J. Barreto, A. Vazquez and E. Camarillo, J. Cryst. Growth, 1989, 96, 685-690.

56 T. Breuer, M. Klues and G. Witte, J. Electron Spectrosc. Relat. Phenomena, 2015, 204, 102-115.

57 B. Meng and W. H. Weinberg, J. Chem. Phys., 1994, 100, 52805289.

58 Individual heating steps took approximately $5 \mathrm{~min}$. Heating was stopped for the NEXAFS measurements, but sample temperatures decreased rather slowly due to the thermal capacity of the sample mount.

59 M. Marks, C. Schmidt, C. H. Schwalb, T. Breuer, G. Witte and U. Höfer, J. Phys. Chem. C, 2012, 116, 1904-1911.

60 E. Habenschaden and J. Küppers, Surf. Sci., 1984, 138, L147L150.

61 M. Polanyi and E. Wigner, Z. Phys. Chem., 1928, 139A, 439452.

62 V. Oja and E. M. Suuberg, J. Chem. Eng. Data, 1998, 43, 486492.

63 L. G. Radchenko and A. I. Kitaigorodskii, Russ. J. Phys. Chem., 1974, 48, 1595-1596.

64 M. Klues and G. Witte, CrystEngComm, 2018, 20, 63-74.

65 J. M. Gottfried, E. K. Vestergaard, P. Bera and C. T. Campbell, J. Phys. Chem. B, 2006, 110, 17539-17545.

66 S. L. Tait, Z. Bohnálek, C. T. Campbell and B. D. Kay, J. Chem. Phys., 2005, 122, 164707.

67 H. Ulbricht, R. Zacharia, N. Cindir and T. Hertel, Carbon, 2006, 44, 2931-2942.

68 J. D. Thrower, E. E. Friis, A. L. Skov, L. Nilsson, M. Andersen, L. Ferrighi, B. Jørgensen, S. Baouche, R. Balog, B. Hammer and L. Hornekær, J. Phys. Chem. C, 2013, 117, 13520-13529.

69 B. Scherwitzl, R. Resel and A. Winkler, J. Chem. Phys., 2014, 140, 184705.

70 B. Scherwitzl, R. Lassnig, M. Truger, R. Resel, G. Leising and A. Winkler, J. Chem. Phys., 2016, 145, 094702.

71 I. Chorkendorff and J. W. Niemantsverdriet, Concepts of Modern Catalysis and Kinetics, Wiley-VCH Verlag GmbH \& Co. KGaA, Weinheim, 2003.

72 A. Redondo, Y. Zeiri, J. J. Low and W. A. Goddard, J. Chem. Phys., 1983, 79, 6410-6415.

73 A. Franco-Cañellas, S. Duhm, A. Gerlach and F. Schreiber, Rep. Prog. Phys., 2020, 83, 066501. 
74 D. G. de Oteyza, I. Silanes, M. Ruiz-Osés, E. Barrena, B. P. Doyle, A. Arnau, H. Dosch, Y. Wakayama and J. E. Ortega, Adv. Funct. Mater., 2009, 19, 259-264.

75 Y. L. Huang, H. Li, J. Ma, H. Huang, W. Chen and A. T. S. Wee, Langmuir, 2010, 26, 3329-3334.

76 Y. Wakayama, D. G. de Oteyza, J. M. Garcia-Lastra and D. J. Mowbray, ACS Nano, 2011, 5, 581-589.

77 E. Goiri, M. Matena, A. El-Sayed, J. Lobo-Checa, P. Borghetti, C. Rogero, B. Detlefs, J. Duvernay, J. E. Ortega and D. G. de Oteyza, Phys. Rev. Lett., 2014, 112, 117602.

78 D. G. de Oteyza, J. M. Garcia-Lastra, E. Goiri, A. El-Sayed, Y. Wakayama and J. E. Ortega, J. Phys. Chem. C, 2014, 118, 18626-18630.

79 P. E. Hofmann, M. W. Tripp, D. Bischof, Y. Grell, A. L. C. Schiller, T. Breuer, S. I. Ivlev, G. Witte and U. Koert, Angew. Chem. Int. E., 2020, 59, 1-6. 